\title{
Depth sensitivity of the dielectric properties of human skin: simulations and measurements
}

\author{
F. Dewarrat, D. Huber, L. Falco-Jonasson, M. S. Talary \\ \& A. Caduff \\ Solianis Monitoring AG, Zürich, Switzerland
}

\begin{abstract}
It has been shown that in vivo glucose level changes affect the dielectric characteristics of the skin and underlying tissue, which can be measured non-invasively by impedance spectroscopy (IS). For a successful implementation of such an impedance sensor it is necessary that the sensor, when placed in contact with human skin, has a sufficient field penetration depth so that it is not only sensitive to changes in the dielectric properties of the upper skin layer, but also has a higher sensitivity to changes in the deeper skin layers. This is due to the fact that the upper layers of the skin are poorly vascularised, therefore their dielectric properties have a low sensitivity to changes associated with blood and intravascular fluids. A simplified model of the human skin and underlying tissue has been developed using electromagnetic field finite element simulations. This model can be used to optimise the electrode design of the impedance sensor. Initially, the applicability of the simulation concepts has been tested by comparing simulations with measurements of reference materials using sensors of various sizes. Subsequently, simulations of the skin model are compared with measurements on human subjects using two experimental procedures. In the first procedure, the blood perfusion of the deeper skin layers is changed by the restriction of the blood outflow (using a pressure cuff). It is shown that the sensitivity to these changes is larger with the sensor with the deeper field penetration, in agreement with simulation results. Similar sensors are then used in a second procedure where the blood glucose concentration is changed. The largest sensitivity to this glucose change is again obtained with the sensor that has the deepest field penetration. Future work will concentrate on quantifying perturbing factors, their effects on the different electrode geometries and the differences between various skin types.
\end{abstract}

Keywords: impedance, skin, non-invasive, glucose, depth profile, simulation. 


\section{Introduction}

Continuous measurement of blood glucose levels has the potential to fulfil requirements of both patients and health care professionals due to its potential to reduce long term effects of diabetes as well as the number of hypoglycaemic episodes. Several minimally and non-invasive techniques for continuous glucose monitoring are currently being developed, one of which is non-invasive impedance spectroscopy (Caduff et al [1]). The sensor used here is a capacitive probe placed in contact with the human skin and measuring the dielectric properties of the skin and the underlying tissue in a defined frequency band (radio frequency, 1-200 MHz). The sensitivity of the measurement to glucose changes is not due to a resonance of the glucose molecule itself, but due to their effect on the dielectric characteristics of blood and tissue (DC and AC conductivity). The electromagnetic field (EMF) generated by the electrode must therefore penetrate deep enough through the upper skin layers which have poor blood perfusion (e.g. the epidermis) and whose dielectric properties are therefore not as much influenced by glucose concentration changes as the deeper tissue layers (e.g. the dermis). However, even if this deep penetration is obtained, the EMF remains to a certain proportion sensitive to the upper skin layer(s) and the impedance measurements are then not only dependent on glucose changes but also on perturbing effects such as changes in upper skin temperature and sweating, for example. Therefore a depth information of the measured signals (e.g. complex impedance) is advantageous for the characterization and later compensation of these perturbing effects. The method proposed here is the use of different electrode sets with different characteristic sizes (i.e. electrode width and distance to ground), ultimately combined with other sensors (such as an optical sensor, temperature sensor, accelerometer) to validate or complete the information required for a feasible glucose monitoring (Caduff et al [2]). This work describes the differences in EMF penetration depth of the different electrode sets as shown by computer simulations, the validation with impedance measurements in vivo on human skin using a procedure with simple physiological changes (sweat changes, skin and tissue perfusion changes) and finally with in vivo impedance measurements using an experimental procedure with defined glucose changes.

\section{Methods}

\subsection{Measurement sensors}

The sensor is made of several electrodes with different characteristic sizes (i.e. width and distance to ground) each surrounded by a ground electrode. In the following description, the electrode with the characteristic size of $4 \mathrm{~mm}$ is called the long electrode and the one with the characteristic size of $0.2 \mathrm{~mm}$ is called the short electrode, referring rather to the EMF penetration than to the electrode length. The electrodes are connected to a vector network analyser (VNA, Rohde \& Schwartz ZVCE) operating between 1 and $200 \mathrm{MHz}$. The VNA measures the 
complex reflection coefficient $S_{I l}$, the complex impedance or admittance, which can be converted with the standard formula $\left(Z=Z_{0}\left(1+S_{11}\right) /\left(1-S_{11}\right)\right.$ and $Y=1 / Z$ where $Z_{0}$ is the reference impedance). Other sensors are used to confirm the physiological changes induced during the procedures. One is an optical sensor composed of a light source placed in contact with skin and a photometer to measure the light reflected by the skin and the underlying tissue. The source emits at a wavelength of $550 \mathrm{~nm}$, which is known to be an adsorption peak of the haemoglobin (Douven and Lucassen [3]), thus providing a measure related to blood perfusion. Another sensor is a sweat sensor measuring the conductance of the skin surface via an interdigitated electrode at low frequency $(1 \mathrm{kHz})$, whose conductance is closely related to the local sweating of the patient. Other sensors are also used such as a temperature sensor or an accelerometer but since their results are not shown here, they are not further described. During the whole procedure including glucose changes, glucose is measured with a HemoCue Glucose 201 Analyzer (HemoCue AB, Sweden).

\subsection{Simulation}

The output of the simulation is the calculation of the EMF generated by the impedance sensor placed in contact with the human skin. The method chosen here is finite element simulation using the commercially available software of Comsol. As explained in a previous work (Talary et al [4]), some simplifications are adopted, i.e. the reduction of the 3-dimensional space to 2-dimensional (crossline perpendicular to sensor length) and the validity of the quasi-static equation for an harmonic potential

$$
-\nabla \cdot\left(\left(\sigma+i \omega \varepsilon_{0} \varepsilon_{r}\right) \nabla V\right)=0,
$$

where $\nabla$ is the nabla operator, $\sigma$ the electric conductivity, $\omega$ the applied field angular frequency, $\varepsilon_{0}$ the dielectric constant, $\varepsilon_{r}$ the relative permittivity and $V$ the electric potential.

The skin is described as a succession of layers parallel to the surface with given thicknesses and dielectric parameters. In a first assumption, each layer is considered as homogeneous in all directions, which obviously does not really reflect the reality since the interface between skin layers is known to be more intricate than a simple plane surface. There are some other physiological features that cannot be described by this simple 2-dimensional model, such as the vertical sweat ducts for example. The frequency dependency is also an important component of dielectric measurements of biological materials but in a first approximation the simulations are run at a single frequency to avoid the difficulty of the dispersion description of each skin layer. This will be the part of further work.

As shown by eqn (1), the physical quantity containing the information is the electrical potential from which the other quantities can be calculated. The physical quantity obtained by a measurement is the complex admittance $Y$ given by: 


$$
Y=G+i \omega C,
$$

where $G$ is the electric conductance (proportional to the electrical conductivity $\sigma$ ) and $C$ the capacitance (proportional to the relative permittivity $\varepsilon_{r}$ ).

The simulation program gives the possibility to calculate the admittance measured by the device, but also the specific part of each layer. This is done by integrating the electric energy or the resistive heating (averaged over a period) over the different skin layer volumes (or surface since it is a 2 dimensional problem):

$$
W_{e l}=\frac{1}{2} C V^{2}, Q_{\text {joule }}=V^{2} G,
$$

where $W_{e l}$ is the stored electric energy (proportional to $\varepsilon_{r} E_{0}{ }^{2}, E_{0}$ is the amplitude of the electric field) and $Q_{\text {joule }}$ the energy dissipated in heat (also called heatloss, proportional to $\sigma E_{0}{ }^{2}$ ). Using the energy conservation principle, according to which the energy stored and dissipated by the total system is equal to the sum of energies stored and dissipated by each layer, i.e.

$$
\varepsilon_{\text {bulk }} \int_{V_{\text {tot }}} E_{0}{ }^{2} d V=\sum_{i}\left(\varepsilon_{L_{i}} \int_{V_{L_{i}}} E_{0}{ }^{2} d V\right),
$$

and

$$
\sigma_{\text {bulk }} \int_{V_{\text {tot }}} E_{0}^{2} d V=\sum_{i}\left(\sigma_{L_{i}}\right) \int_{V_{L_{i}}} E_{0}^{2} d V,
$$

respectively (where $\mathrm{V}_{\text {tot }}$ is the total volume, $\mathrm{V}_{\mathrm{Li}}$, the volume of the i-th layer with dielectric parameters $\varepsilon_{\mathrm{Li}}$ and $\sigma_{\mathrm{Li}}$ ), one can show that the contribution of certain layer to the bulk dielectric parameters $\varepsilon_{b u l k}$ and $\sigma_{b u l k}$ is proportional to the energy stored and dissipated by this layer. Therefore the cumulative plot of the electric energies or heatlosses for each skin layer divided by the total electric energy or heatloss shows the relative contributions of the skin layer to the measured capacitance or conductance. This way of showing the relative contribution of the different layers to the total capacitance or conductance gives similar results to the case where the relative changes of capacitance and conductance versus changes of layer permittivity or conductivity are calculated. Once the conductance and the capacitance are known, it is possible to calculate the conductivity and relative permittivity of the measured material as seen by the sensor with the calibration parameters obtained with measurements on known reference materials.

The skin model used for the simulations comprises the following layers. The first layer represents the mixture of the sebum that is found on the surface of the skin and a sweat layer due to the fact that under the sensor, the water emitted by the skin cannot evaporate. Therefore its conductivity $(0.4 \mathrm{~S} / \mathrm{m})$ and relative 
permittivity (75) are rather large. The two next layers, stratum corneum and epidermis, represent the upper skin, which has low blood perfusion and low water content, with conductivity ranging from millisiemens per meter to several centisiemens per meter and relative permittivity below twenty. The deeper skin layers, dermis, fat and muscle, have a larger water content and a larger blood perfusion, which means higher conductivity (up to 0.7 Siemens per meter) and relative permittivity (up to 110), with the exception of fat (conductivity in tens of millisiemens, relative permittivity below twenty). These parameters come from a combination of different literature resources (Gabriel et al [5], Martinsen and Grimnes [6], Martinsen et al [7], Nicander and Ollmar [8]) or estimated in accordance to their blood or water content for the cases where the values could not be explicitly found. Since the questions raised in this article concern general considerations but not detailed comparisons with absolute values of measured quantities, we do not discuss here the influence of other parameter sets on the results. An analysis of the variations between patient skin and measured results will be done in another work.

\subsection{Study protocols}

Two different experimental/clinical studies have been performed. The first one contains procedures where different perturbations are induced on the upper (sebum/sweat, stratum and epidermis) and lower (dermis, fat and muscle) skin layers. A period of $15 \mathrm{~min}$ of physical activity on a home trainer lets the patient sweat, which mainly affects the dielectric properties of the upper skin layers. Skin perfusion changes are induced with a pressure cuff placed on the upper arm that reduces for several minutes the blood flow in the veins. At that point the blood amount in the deeper skin layers increases and changes their dielectric properties. A detailed description of the study protocol and results can be found in reference Talary et al [9].

The second experimental/clinical study contains procedures where the blood glucose levels are changed to follow a predefined profile using a manual intravenous glucose clamp technique as described by Heinemann and AmpudiaBlasco [10]. The glucose profile features two hyperglycemic excursions. Again more details can be found in reference Caduff et al [2].

\section{Results}

\subsection{Simulation}

Figure 1 shows the relative part of electric energy and heatloss in the different skin layers for the long and short sensors. The relative parts of the electric energy and heatloss deposited in upper skin layers (i.e. sebum, stratum and epidermis) is around two and four times larger for the short sensor than for the long sensor. Similarly, the relative parts of the electric energy and heatloss deposited in the deeper skin layers (dermis, fat and muscle) are both around five times larger for the long sensor than for the short sensor. These observations are valid for both 
electric energy and heatloss, but the respective parts are still different. For example there is no heatloss deposited in the sensor substrate (base) since its conductivity is almost zero. Since the conductivities of the skin layers cover a broader range than the permittivities, the relative part of heatloss is more extreme than the one of electric energy (the relative heatloss of the epidermis seen by the short sensor is very large, as well as the cumulative of dermis, fat and muscle by the long sensor).
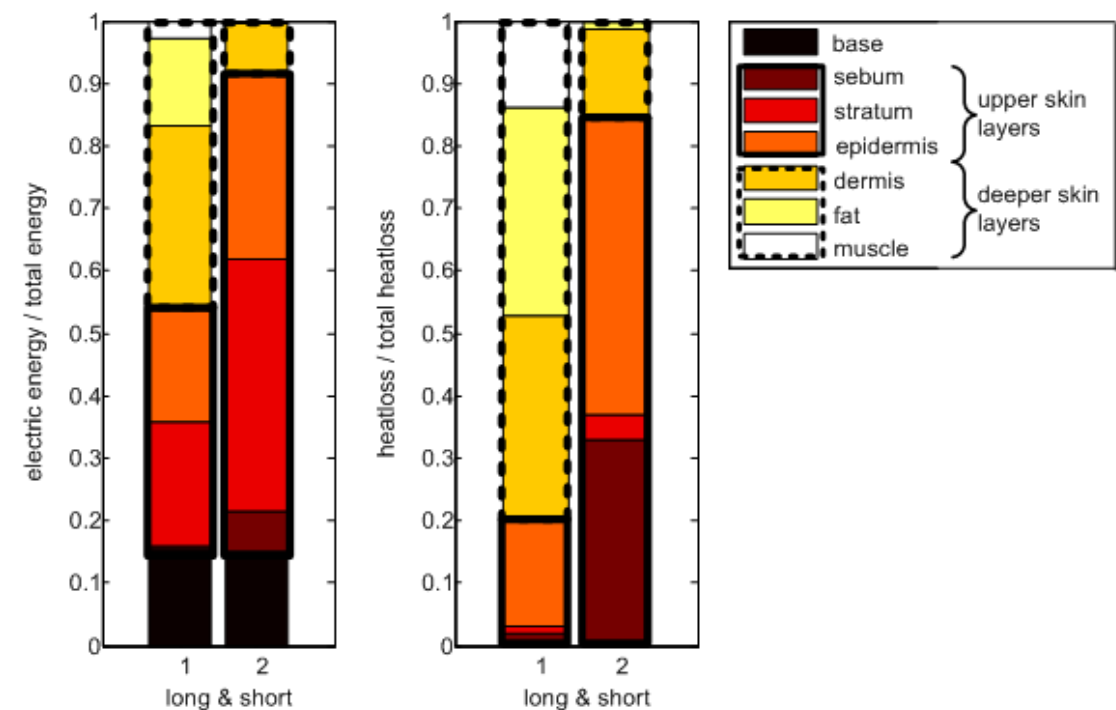

Figure 1: Relative electric energy (left) and heatloss (right) in the sensor base and in the different skin layers for the electrodes of characteristic size $4 \mathrm{~mm}$ (long) and $0.2 \mathrm{~mm}$ (short). The skin layers are grouped in upper and deeper skin layers as explained in text.

\subsection{Measurements}

As described above, the first part of the measurements consists of applying defined procedures in order to change dielectric properties of upper and deeper skin layers separately.

The measurement examples shown in fig. 2 qualitatively agree with simulation results. The relative changes of the conductance and the capacitance during blood perfusion changes are larger for the measurement done with the long sensor (fig. $2 \mathrm{a}$ and $\mathrm{b}$ ) than for the one done with the short sensor, which is almost insensitive to these changes (fig. 2 c). On the contrary, the relative change of the conductance measured by the short sensor is large during the sweat event and almost zero for the long sensor. A sweat sensor measuring the surface conductance at low frequency (fig. 2 c) validates the interpretation of the short sensor signal. The measurement of the optical reflection at a wavelength of 550 
$\mathrm{nm}$ (fig. $2 \mathrm{~d}$ ) confirms the increase of skin perfusion, since the presence of more blood in the measured skin volume results in a higher absorption and hence a lower optical reflection (Douven and Lucassen [3]). For this study day the sensitivity of the long impedance sensor to sweat is almost zero (fig.2 a and b), but this is not always the case, therefore the presence of a short sensor is beneficial.

a)

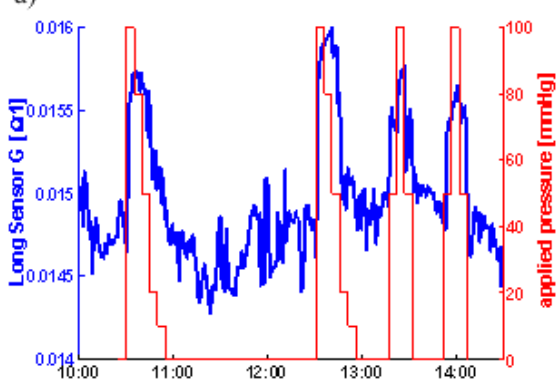

c)

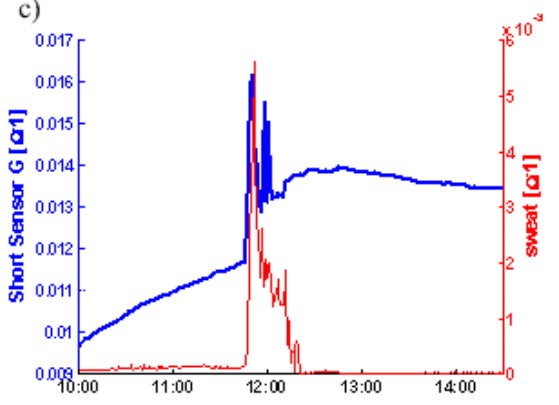

b)

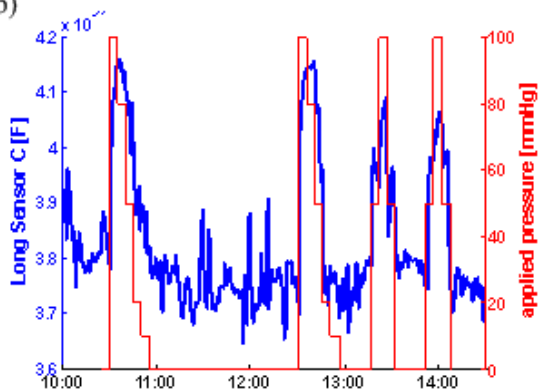

d)

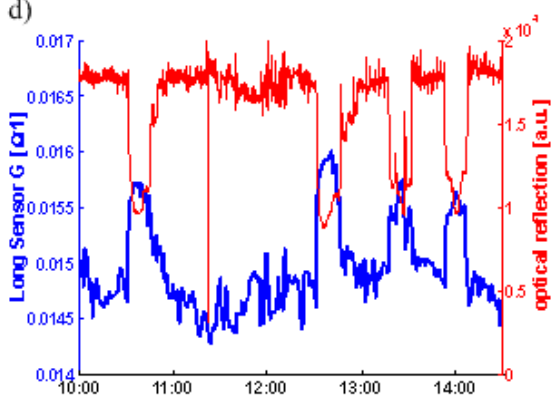

Figure 2: Results of one test day: a) Comparison of conductance (G, bold line) measured with the long sensor and the applied pressure (thin line); b) capacitance (C) measured with the long sensor and applied pressure; c) conductance measured with the short sensor and sweat; d) conductance measured with the long sensor and optical reflection. The measurements are plotted towards the time.

The second clinical study aimed to show the admittance response during procedures involving glucose changes, and several electrode sets with different characteristic sizes were used as shown in the following graph.

As stated in the introduction, the glucose concentration changes induce changes on the dielectric properties of the blood and the tissue. The results shown in fig. 3 are in agreement with previous results and with the physiological location of the glucose changes. The sensitivity of the conductance (i.e. the amplitude of the standardised quantity) to glucose changes increases with increasing electrode characteristic sizes, since this information is mainly contained by the blood, which is a minor component of the upper skin layers. It 
was observed on fig. 2 that the sensitivities of conductance and capacitance to perfusion were of similar order of magnitude. The fig. 3 shows that it is not fully verified for the case where the blood glucose concentration is changing, indicating that the permittivity changes induced by glucose are of a more complex nature than simple perfusion changes.

The results of fig. 3 should however not be misinterpreted. The conductance and capacitance measured with the electrode with $4 \mathrm{~mm}$ characteristic size are not the one of the deep skin layers only, but the one of each skin layer with relative contribution as shown in fig. 1. The task of determining the specific dielectric values for each separated layer based on the measurements of the electrodes with various sizes will be done in a further work.

a)

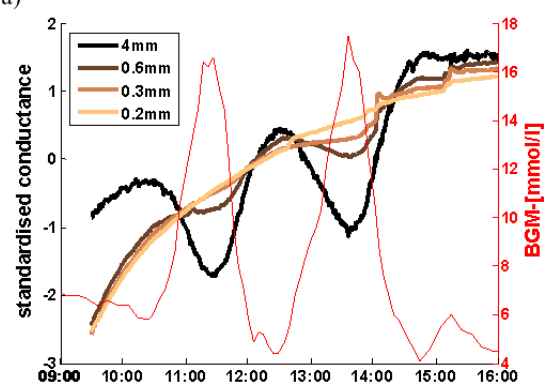

b)

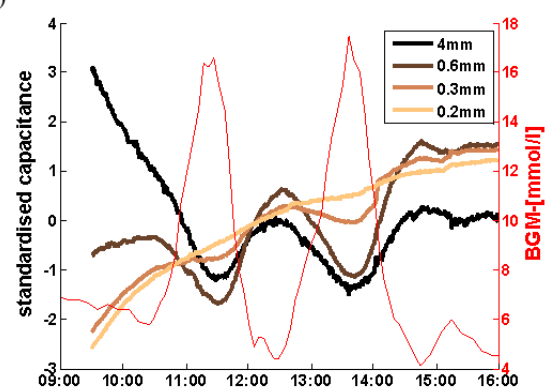

Figure 3: $\quad$ Comparison of standardised $\left(\left(\mathrm{G}-\mathrm{G}_{\text {mean }}\right) / \mathrm{G}_{\text {stdeviation }}\right)$ conductance (a) and capacitance (b) for various electrode characteristic sizes (thick lines) with blood glucose (BGM, thin line) on one study day. The measurements are plotted versus time.

\section{Conclusions}

This work illustrates the differences in the depth sensitivity of dielectric parameters obtained with sensors of different characteristic sizes using a simplified skin model. The distribution of the simulated energies in different skin layers shows how the contribution of deeper skin layers increase with increasing electrode size. The simulations are validated with experimental results where changes in upper or deeper skin layers are induced, namely via an increase of sweat on the skin surface or changes in skin perfusion. As expected, the short sensor has a clear sensitivity to the skin surface changes and the long sensor a clear sensitivity to the deeper tissue changes. Then similar sensors are used to measure skin admittance during glucose excursions, and again a higher sensitivity is obtained with the sensor having larger penetration depth. Further work will be performed to simulate the frequency dependency of the admittances and compare them with in vivo measurements. Ultimately, these signals are used to allow for a glucose related calculation under daily life conditions as part of a multi sensor platform. 


\section{References}

[1] Caduff, A., Dewarrat, F, Talary, M., Stalder, G., Heinemann, L. \& Feldman, Y., Non-invasive glucose monitoring in patients with diabetes: A novel system based on impedance spectroscopy, Biosensors and Bioelectronics, 22(5), pp. 598-604, 2006.

[2] Caduff, A., Donath, M., Talary, M., Haug, S., Huber, D., Stahel, W. A, Dewarrat, F., Jonasson, L.S., Krebs, H.-J. \& Klisic J, Multisensor concept for non-invasive physiological monitoring, Proc. of IMTC, in press.

[3] Douven, L. F. A. \& Lucassen, G. W., Retrieval of optical properties of skin from measurement and modelling the diffuse reflectance, Proc. of Laser-Tissue Interaction XI: Photochemical, Photothermal and Photomechanical, eds. D. D. Duncan, J. O. Hollinger \& S. L. Jacques, SPIE vol 3914, pp. 312-323, 2000

[4] Talary, M. S., Dewarrat, F., Caduff, A., Puzenko, A., Ryabov, Y. \& Feldman, Y., An RCL Sensor for Measuring Dielectrically Lossy Materials in the $\mathrm{MHz}$ Frequency Range 1. Comparison of Hydrogel Model Simulation with Actual Hydrogel Impedance Measurements, IEEE Transactions on Dielectrics and Electrical Insulation, 13(2), pp. 247-256, 2006.

[5] Gabriel, S., Lau, R.W. \& Gabriel, C., The dielectric properties of biological tissues: II. Measurements in the frequency range $10 \mathrm{~Hz}$ to 20 GHz, Phys. Med. Biol., 41, pp. 2251-2269, 1996.

[6] Martinsen, O.G. \& Grimnes, S., Facts and myths about electrical measurements of stratum corneum hydration state, Dermatology, 202, pp. 87-89, 2001.

[7] Martinsen, O. G., Grimnes, S. \& Schwan, H. P., Interface phenomena and dielectric properties of biological tissue, Encyclopedia of Surface and Colloid Science, ed. A. T. Hubbard, pp. 2643-2652, 2002

[8] Nicander, I. \& Ollmar, S., Electrical bioimpedance related to structural differences and reactions in skin and oral mucose, Ann. N. Y. Acad Sci, 873, pp.221-226

[9] Talary, M., Dewarrat, F., Huber, D. \& Caduff, A., In vivo life sign application of dielectric spectroscopy and non-invasive glucose monitoring, Journal of Non-Crystalline Solids, in press

[10] Heinemann, L. \& Ampudia-Blasco, F. J, Glucose clamps with the Biostator: a critical reappraisal, Horm. Metab Res., 26(12), pp. 579-583, 1994. 